\title{
CS-17 Composición de oligoelementos de hojas comestibles nativas de Mesoamérica
}

\author{
Trace element content in edible leaves natives in Mesoamerica \\ Armando Cáceres ${ }^{1,3 *}$, Vicente Martínez², Max Mérida ${ }^{1,3}$, Anibal Sacbajá ${ }^{2}$, \\ Alejandra Morales ${ }^{1}$, Sully M. Cruz ${ }^{1}$
}

${ }^{1}$ Facultad de Ciencias Químicas y Farmacia y ${ }^{2}$ Facultad de Agronomía, Universidad de San Carlos de Guatemala y ${ }^{3}$ Laboratorios de Productos Naturales Farmaya, Guatemala.

*Autor al que se dirige la correspondencia: acaceres46@gmial.com

\section{Resumen}

L os oligoelementos son importantes constituyentes nutricionales de las hierbas comestibles. Se colectaron 11 - especies, nueve nativas (Amaranthus hybridus, Cnidoscolus aconitifolius, Crotalaria longirostrata, Dysphania ambrosioides, Lycianthes synanthera, Sechium edule, Solanum americanum, S. nigrescens, S. wendlandii) y dos extranjeras (Moringa oleifera, Spinacea oleracea) en dos regiones del país. Se prepararon muestras de hierba seca, cocida y de caldo de hierba fresca, en las que se cuantificaron por espectrofotometría de absorción atómica macro (N, $\mathrm{P}, \mathrm{K})$ y oligoelementos $(\mathrm{Ca}, \mathrm{Mg}, \mathrm{Na}, \mathrm{Cu}, \mathrm{Zn}, \mathrm{Mn})$ y los componentes antinutricionales, taninos por espectrofotometría usando ácido tánico como control y oxalatos por permanganimetría. El contenido de oligoelementos es diverso, para Zn, la hierba control $S$. oleracea contienen buena cantidad (90-140 ppm); de las nativas D. ambrosioides (130-160 ppm) y A. hybridus (70-80 ppm) demostraron la mayor cantidad. De Fe las hierbas control tienen buena composición (S. oleracea, 220-280 ppm y M. oleifera, 105-135 ppm); de las nativas A. hybridus (90-240 ppm), C. aconitifolius $(75-185 \mathrm{ppm})$ y L. synanthera $(75-140 \mathrm{ppm})$ tuvieron las mayores concentraciones. Se encontraron niveles elevados de oxalatos en $S$. oleracea $(722 \pm 6 \mathrm{mg} / \mathrm{hg})$, L. synanthera $(678 \pm 8 \mathrm{mg} / \mathrm{hg})$, S. nigrescens $(455$ $\pm 7 \mathrm{mg} / \mathrm{hg}$ ) y $A$. hybridus (362 $\pm 9 \mathrm{mg} / \mathrm{hg}$ ); en las demás hierbas se encontraron niveles $<90 \mathrm{mg} / \mathrm{hg}$. Los niveles de taninos fueron bajos (0.1-0.7 mg/hg) en todas las especies. Se demuestra que cuatro especies nativas tienen un contenido de oligoelementos importante y que presentan valores antinutricionales menores que los controles.

Palabras claves: Amaranthus hybridus, Cnidoscolus aconitifolius, Lycianthes synanthera

\section{Abstract}

$\mathrm{T}$ race elements are important nutritional constituents from edible herbs. Eleven species were collected, nine native (Amaranthus hybridus, Cnidoscolus chayamansa, Crotalaria aconitifolius, Dysphania ambrosioides, Lycianthes synanthera, Sechium edule, Solanum americanum, S. nigrescens and S. wendlandii) and two foreign (Moringa oleifera Spinacea oleracea) in two regions of the country. Dry, cooked and broth samples were prepared by atomic absorption spectrometry, macro ( $, \mathrm{P}, \mathrm{K})$ and trace elements $(\mathrm{Ca}, \mathrm{Mg}, \mathrm{Na}, \mathrm{Cu}, \mathrm{Zn}, \mathrm{Mn}$ ) were quantified, as well as antinutritional components, tannins by spectrophotometry using tannic acid as control and oxalates by permanganometry. Trace element content is diverse, for $\mathrm{Zn}$, control herb $S$. oleracea contained good quantity (90$140 \mathrm{ppm})$; D. ambrosioides (130-160 ppm) and A. hybridus (70-80 ppm) contained the largest amounts. For Fe, control herbs had good composition (S. oleracea, 220-280 ppm, M. oleifera, 105-135 ppm); from the natives $A$. hybridus (90-240 ppm), C. aconitifolius (75-185 ppm) and L. synanthera $(75-140 \mathrm{ppm})$ had the largest amounts. High levels of oxalates were demonstrated in S. oleracea $(722 \pm 6 \mathrm{mg} / \mathrm{hg})$, L. synanthera $(678 \pm 8 \mathrm{mg} / \mathrm{hg})$, S. nigrescens $(455 \pm 7 \mathrm{mg} / \mathrm{hg})$ and A. hybridus $(362 \pm 9 \mathrm{mg} / \mathrm{hg})$; in the others $<90 \mathrm{mg} / \mathrm{hg}$ were found. Tannin levels were low $(0.1-0.7 \mathrm{mg} / \mathrm{hg})$ in all species. It is concluded that four native species has important trace element content and showed antinutritional element lower than controlers. 